DOI: $\underline{\text { https://doi.org/10.34069/AI/2021.42.06.20 }}$

How to Cite:

Atencio Bravo, E.A., Rojas Hernández, L.M., \& Piñero Landaeta, L. del V. (2021). Competencias del investigador en el contexto tecnológico y su correspondencia con las políticas públicas en ciencia, tecnología e innovación. Amazonia Investiga, 10(42), 217-225. https://doi.org/10.34069/AI/2021.42.06.20

\title{
Competencias del investigador en el contexto tecnológico y su correspondencia con las políticas públicas en ciencia, tecnología e innovación
}

\section{Researcher competencies in the technological context and its correspondence with public policies in science, technology and innovation}

Written by:

Eduardo Alfonzo Atencio Bravo ${ }^{90}$ https://orcid.org/0000-0003-0294-5289 Lucia Magdalena Rojas Hernández ${ }^{91}$ https://orcid.org/0000-0003-4416-9206

Liliana del Valle Piñero Landaeta ${ }^{22}$ https://orcid.org/0000-0002-1767-5106

\section{Resumen}

Este estudio presenta el análisis de las competencias del investigador en el contexto tecnológico y su correspondencia con las políticas públicas venezolanas de ciencia, tecnología e innovación. Metodológicamente, se asumió un tipo de investigación analítica mediante un diseño documental bibliográfico, tomando como referencia los perfiles de competencias generales de instituciones universitarias públicas y privadas de la Subregión Costa Oriental de Lago-Estado Zulia, al igual que las disposiciones regulatorias del Sistema Nacional de Ciencia, Tecnología e Innovación. La información se recopiló mediante la técnica de revisión documental apoyada en una matriz de registro como instrumento, dando lugar al uso de la técnica de análisis de contenido para el establecimiento de categorías y subcategorías emergentes del proceso efectuado desde una perspectiva hermenéutica cualitativa. Los resultados permitieron concluir que existe una desarticulación entre las competencias investigativas prescritas en los perfiles de egreso y las políticas públicas estudiadas, lo que indica la necesidad de formular criterios de desempeño orientados al fortalecimiento de las capacidades nacionales de CTI directamente

\begin{abstract}
This study presents the analysis of the researcher's competences in the technological context and its correspondence with the Venezuelan public policies of science, technology and innovation. Methodologically, a type of analytical research was assumed through a bibliographic documentary design, taking as a reference the profiles of general competencies of public and private university institutions of the Eastern Coast Sub-region of Lake-Zulia State, as well as the regulatory provisions of the System National Science, Technology and Innovation. The information was collected using the document review technique supported by a registration matrix as an instrument, giving rise to the use of the content analysis technique for the establishment of emerging categories and subcategories of the process carried out from a qualitative hermeneutical perspective. The results allowed to conclude that there is a disarticulation between the investigative competencies prescribed in the graduate profiles and the public policies studied, which indicates the need to formulate performance criteria aimed at strengthening national STI capacities directly from the intentions declared in the university curriculum, specifically for engineer training.
\end{abstract}

\footnotetext{
${ }^{90}$ Postdoctor en procesos de la ciencia y la investigación. Doctor en Ciencias mención Gerencia. Universidad Euroamericana. Panamá.

${ }^{91}$ Doctora en Ciencias Gerenciales. Universidad del Sinú. Colombia. Universidad Euroamericana. Panamá.

92 Doctora en Ciencia de la Educación. Universidad Euroamericana. Panamá.
} 
desde las intencionalidades declaradas en el currículo universitario, específicamente para la formación del ingeniero.

Palabras clave: Perfil de competencias, investigador tecnológico, políticas públicas, ciencia, tecnología e innovación.

\section{Introducción}

La precipitada trasformación digital que se experimenta en la aldea global, ha develado la necesidad de trascender a nuevos patrones tecnocientíficos que procuren explicar, comprender y transformar la dinámica de la realidad, donde el desarrollo de las competencias investigativa juega un papel preponderante frente a la resolución de las complejidades que dibujan la coyuntura social, económica, política, educativa, científica y cultural de estos tiempos. De tal manera, se asume la formación del recurso humano en investigación como eje medular para el incremento del capital intelectual con base a las capacidades y recursos que son condicionantes para la generación y transferencia del conocimiento desde el seno de las instituciones de educación superior, eventualidad que apunta a la formación integral del investigador en alineación con las necesidades de desarrollo en el campo de la ciencia, tecnología e innovación, según las prioridades de cada país o región.

Siendo así, el esfuerzo estratégico de gran parte de las universidad de la latitud por desarrollar el pensamiento crítico del estudiante desde la formación investigativa, pero además, incrementar la producción y visibilidad de la investigación en términos de la inversión pública, los planes nacionales de ciencia, tecnología e innovación, las miradas y diálogos conjuntos que configuran mecanismos de acción, y no obstante, los estímulos que promueven la investigación, desarrollo e innovación $(\mathrm{I}+\mathrm{D}+\mathrm{I})$, ha sido una tarea impostergable desde el anhelo de un aparato socioprouctivo nacional en constante crecimiento desde la inventiva, desarrollo, transferencia e innovación tecnológica, y consecuentemente, el escalamiento industrial que desde programas curriculares de naturaleza aplicada encuentran una marcada acentuación.

Sin embargo, la formación del talento humano en investigación y su vinculación con las Actividades de Ciencia, Tecnología e Innovación (ACTI) desde los escenarios de la educación superior, cada día resultan un nudo crítico en lo que respecta formación, desarrollo y transferencia de nuevos procesos, productos y/o
Keyword: Skills profile, technological researcher, public policies, science, technology and innovation.

servicios tecnológicos, convirtiéndose de tal manera en una situación que convoca a centrar la mirada en la calidad de los procesos formativos curriculares y su pertinencia frente a la realidad nacional que dictamina la política nacional en Ciencia, Tecnología e Innovación, y el mercado ocupacional al cual se insertará el futuro profesional.

Lo anterior, se argumenta desde los planteamientos de Atencio (2017), quien precisa según la Red Iberoamericana e Interamericana de Ciencia y Tecnología (RICyT) que:

En América Latina y el Caribe para el año 2012, se ha asumido una inversión en actividades de Ciencia, Tecnología e Innovación (CTI) en relación al Producto Interno Bruto (PBI) del $1,15 \%$, destinando a su vez un $0,74 \%$ para las actividades de Investigación y Desarrollo (I+D). Sumado a ello, para el año antes mencionado el continente tratado presenta un total de 382.961 investigadores; 95.690 becarios de I+D / Doctorado, y 21.275 patentes otorgadas, cifras que denotan un alto interés por la gestión pública ante el campo tecno-científico, aun cuando la $\mathrm{I}+\mathrm{D}$ demanda mayor infraestructura. (Atencio, 2017)

Desde este marco estadístico se logra evidenciar que los gobiernos de los países de Latinoamérica están evolucionando drásticamente en la formulación y desarrollo de políticas, instrumentos e instituciones que fortalezcan las capacidades nacionales en el sector de la ciencia, tecnología e innovación, y una muestra de ello lo constituye el (Decreto $\mathrm{N}^{\circ} 1411,1986$ ), publicado en Gaceta Oficial Extraordinaria $\mathrm{N}^{\circ} 6.151$, quien mediante su artículo 4, expresa que:

"la autoridad nacional con competencia en materia de ciencia, tecnología, innovación y sus aplicaciones debe formular la política pública nacional de ciencia, tecnología, innovación y sus aplicaciones, basada en el Plan Nacional de Desarrollo Económico y Social de la Nación, la sustentabilidad de la producción...” (p. 16) 


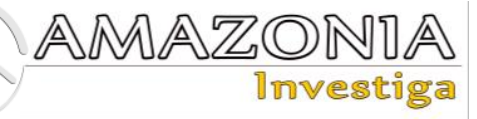

Según Atencio (2014), hoy día, cuando se le ha asignado mayor relevancia a los procesos vinculados con la ciencia, tecnología e innovación, cobra importancia la formación del talento humano, espacio que tal vez se podrá visibilizarse desde el desarrollo de competencias en quienes hacen vida académica en los diversos recintos universitarios, mediante modelos curriculares adaptados a las necesidades del entorno, que realmente respondan a las necesidades de investigación definidas por el Ministerio del Poder Popular para la Ciencia y Tecnología de Venezuela (2011), entre las cuales se encuentran: ambiente, energía, telecomunicaciones, política, sociedad, educación, vivienda, hábitat y desarrollo urbano, salud colectiva $y$ finalmente seguridad $y$ soberanía alimentaria.

Al respecto, Bozo de Carmona, Camacho, Carillo, Casilla, Inciarte, Morales, Rincones, y Parra (2009), refieren que en algunas universidades del contexto venezolano se ha dado inicio al diseño curricular de perfiles académicos profesionales desde el enfoque por competencias, como producto de la incorporación del Proceso de Bolonia, el cual planteó la creación de un sistema de grados académicos flexibles y comparables desde el reconocimiento de estudios, el fomento de la movilidad estudiantil, docente e investigativa, en búsqueda de lograr la calidad de la enseñanza e incursionar en la propuesta del Espacio Europeo de Educación Superior, fundamentalmente por influencia de la difusión del Proyecto Tuning en Venezuela en el año 2000, cuyas implicaciones se inclinan al desarrollo de titulaciones comparables y comprensibles que contribuyan a la inserción de todo estudiante o profesional en el mundo académico y/o laboral.

En este orden de ideas, las referidas autoras señalan que los modelos curriculares de las universidades nacionales han incorporado el enfoque por competencias reconociendo las repercusiones del mismo en la concepción, práctica y evaluación de la formación profesional, partiendo de las líneas decretadas en la declaración y proyecto antes citado, pero es preciso señalar que los esfuerzos que han hecho las universidades con relación al currículo por competencias se encuentran en su mayoría en la fase de diseño.

Por lo antes expuesto, es imperante la necesidad de orientar el diseño curricular bajo el enfoque competencial centrado en la investigación, el cual según Perilla (2018),
Corresponde a un proceso de construcción que debe trascender las dinámicas de imposición, para asegurar pertinencia desde las características de cada contexto específico. De este modo, antes de diseñar un currículo deben ser identificados los antecedentes del mismo y ellos pueden estar delimitados desde un nivel macro hasta un nivel micro; evitando de esta manera empezarlo desde la perspectiva de qué hacer, para centrarse en un para qué encaminado a dotar de utilidad la formación. (P.34)

En el caso particular de las instituciones universitarias de carácter públicas o privadas del estado Zulia, específicamente de la Subregión Costa Oriental del Lago de Maracaibo, estas han orientado la transformación curricular de sus programas académicos mediante el abordaje del mencionado enfoque de competencias, donde la investigación, innovación y el desarrollo, suelen considerarse como competencias genéricas $o$ generales que transversalizan el proceso de formación profesional en todos sus componentes, proporcionando al estudiante universitario, herramientas, conocimientos y valores necesarios para la transformación de la realidad mediante procesos investigativos, con miras a la solución de problemas prácticos y la producción de conocimientos científicos y tecnológicos.

Sin embargo, se debe acotar que dichas competencias centradas en la investigación, tal vez se encuentran desarticuladas de las exigencias políticas nacionales en materia de ciencia, tecnología e innovación, debido quizás a debilidades y falta de énfasis en los procesos para el desarrollo e innovación tecnológica. Sumado a ello, resulta que tales competencias carecen de pertinencia y adecuación a las situaciones actuales que demanda el entorno, en lo que respecta a procesos de inventiva popular, escalamientos industriales, generación de prototipos, registros de patentes, entre otros aspectos que demandan un currículo universitario pertinente y centrado en la investigación, el desarrollo y la innovación, fundamentados en perfiles académicos cónsonos a la realidad del contexto y de la profesión. De allí que la postura curricular que aboga por un perfil de competencias investigativa recobra fuerzas ante la congruencia de la formación profesional que se amerita en el campo tecnológico, cuyos espacios formativos se deben al desarrollo de tales competencias, sin desvirtuar las intencionalidades curriculares que terminan promoviendo el desarrollo de procesos investigativos conforme a los parámetros de otras ciencias como las del campo humanístico social, que si bien es cierto, son complementarias ante la 
resolución de problemas prioritarios que requieren capacidad nacionales de ciencia, tecnología e innovación.

Es por todo lo anterior que el presente estudio se centró en analizar las competencias del investigador en el contexto tecnológico y su correspondencia con las políticas públicas venezolanas de ciencia, tecnología e innovación, con el fin de responder la siguiente interrogante: ¿En qué medida se corresponden las competencias investigativas del futuro profesional del contexto tecnológico con las políticas públicas de ciencia, tecnología e innovación, y la resolución de problemas del contexto social venezolano?.

\section{Revisión de literatura}

\section{Currículo universitario, investigación y políticas públicas}

Comprender la dinámica curricular y los cambios vertiginosos que supone la formación profesional en contextos caracterizados por la incertidumbre, representa un reto para quienes intervienen en la ardua tarea de responder a los compromisos para con la sociedad actual, cuyas formas de interactuar, construir el conocimiento, comunicarse, y por sobre todo, de aprender han cambiado exponencialmente, promoviendo nuevas estructuras con base a la investigación y la articulación flexible entre teoría-experiencia, dando lugar ecosistemas de formación universitaria imaginables. No obstante, parece ser una lección no aprendida que el currículo es mucho más que simples contenidos normativos, y que estos encuentran pertinencia y esencialidad en la medida de la significatividad que le otorgue el estudiante, y desde ella, logre transferirlo a partir de la integralidad de saberes que propenden al desempeño idóneo esperado frente a la resolución de problemas complejos. En ese sentido, según Atencio (2014).

El currículo como sistema complejo y dialógico de articulación sociocultural entre la universidad y el contexto que lo rodea, ha trascendido los diversos enfoques pedagógicos que pretenden reinventar la praxis educativa, partiendo del hecho didáctico mediante un conjunto de componentes cónsonos para su propio diseño, el cual busca la integralidad de saberes (conocer, ser, hacer, convivir y emprender), para la formación pluridimensional del ser humano, tomando en cuenta la concepción de hombre a formar, las normativas de diseño curricular vigentes, y por ende las necesidades del entorno, a las cuales se busca responder con el desempeño idóneo de profesionales que demuestren competencias y disposición ética para el afrontamiento de la vida, mediante la vinculación con todos los actores que hacen vida académica en los recintos universitarios (2014).

En consonancia a lo anterior, Inciarte y Canquiz (2008), refieren que uno de los retos fundamentales, que ya se han venido delineando es la integralidad en la formación, la cual solo será posible en la medida que se dé la pertinencia, entendida esta como la repuesta filosófica, científica, social, personal, laboral e institucional. En este sentido, las autoras plantean que para que se haga realidad dicha concepción, la acción debe orientar el currículo hacia la satisfacción de expectativas del contexto social, científico e institucional, además de la congruencia interna de los elementos estructurales.

En el mismo orden de ideas, para las autoras, tanto la integralidad como la pertinencia hacen exigencias a los actores fundamentales del currículo, los cuales deben reconocer que la educación no representa una serie de aprendizajes definitivos, sino una búsqueda permanente, en la que todo tiene un sentido y puede cambiar; por lo que entonces el aprendizaje, el egresado, el conocimiento, la tecnología, como productos del currículo, no son productos acabados sino en continua generación y transformación.

Desde la postura de los investigadores, el currículo se conceptualiza como un macrosistema que argumenta la concreción del proceso formativo en todas sus instancias, atendiendo a los planteamientos de un marco filosófico, unas concepciones originarias de hombre, sociedad, cultura y educación, y un marco teórico curricular sobre el cual se edifican las intencionalidades del diseño curricular, y desde este, el plan de estudio, perfiles académicos profesionales, programación analítica y un conglomerado de instrumentos metodológicos que idóneamente buscan responder a las demandas de la sociedad y el mercado ocupacional, sumado a las necesidades detectadas frente a la formación profesional y las competencias técnicas y humanas que este requiere para el mundo globalizado. En consonancia a dicho planteamiento, Castillo y Cabrerizo (2006), precisan que el diseño curricular, se concibe como:

Un proceso dinámico y estático que genéricamente se concibe como el conjunto de pautas, orientaciones o prescripciones que, 


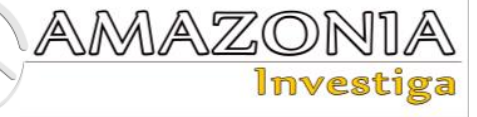

estructurados en fases, tienen como fin elaborar o diseñar la enseñanza de una determinada materia, área o asignatura universitaria, considerando las necesidades sociales, la práctica educativa, el ejercicio profesional y los sujetos de la instrucción-formación.

Por su parte, Tobón (2010), señala que el diseño del currículo desde el enfoque socioformativo, busca implementar estrategias que faciliten en todos los miembros de la institución educativa un modo de pensamiento complejo, basados en aspectos esenciales tales como la autorreflexión, la autocrítica, la contextualización del saber, la multidimensionalidad de la realidad, la comprensión de aquello que se quiere conocer e intervenir, y el afrontamiento estratégico de la incertidumbre.

En ese marco de reflexión, el diseño curricular bajo las aproximaciones del enfoque tratado por Tobón, promueve que los integrantes de la comunidad educativa se formen de manera plena, con un proyecto ético de vida sólido, emprendimiento creativo y competencias, con base en el apoyo de cuatro principios clave del pensamiento complejo: auto-eco-organización, recursividad organizacional, dialógica y hologramática, los cuales se ponen en acción a través de ciclos propedéuticos, proyectos, prácticas y pasantías.

Volviendo la mirada a la concepción del currículo como sistema complejo, se entiende que éste, ha de estar constituido por diversos componentes curriculares, los cuales son indispensables para abordar su confección. Desde este punto de vista, la concreción del dicho diseño se debe asumir dialógica y reflexivamente bajo la ruta que el curricultor diseñe o adopte, y, que considere pertinente para el logro de un currículo que se corresponda con las vigencias del entorno social, desde el delineado de competencias congruentes y pertinentes con los planes de ciencia, tecnología e innovación que apalancan el desarrollo del país y la sociedad en general.

Sobre este escenario, Rincones (2007), plantea que el transcurrir del diseño curricular responde a tres estadios.

El primero de ellos, denominado Macrocurriculo, el cual se orienta al diagnóstico contextual de la institución como proyecto organizacional, la definición de ser humano que se pretende formar, y la presentación y justificación del modelo teórico curricular que se asumirá. En segunda instancia, se tiene el Mezzocurriculo, centrado en la elaboración de perfiles académicos profesionales, plan de estudio y malla curricular con todos sus indicadores cuantitativos. Finalmente, la autora asume el Microcurriculo, como instancia para elaborar los programas formativos de cada unidad curricular concebida en el plan de estudios, precedido de las estrategias formativas y de evaluación.

Ahora bien, para quienes abordan el hecho del diseño curricular bajo el enfoque de formación humana basado en competencias, debe estar claro que dichas competencias son consideradas como dispositivos de articulación de saberes que orientan al individuo a un desempeño basado en el mejoramiento continuo, contribuyendo a solventar problemas del contexto con responsabilidad social y compromiso éticomoral. Dado estas características, todo perfil académico profesional contentivo de las competencias genéricas y específicas a demostrar por el futuro profesional, debe inducir a un proceso formativo a fin de consolidar los conocimientos, actitudes, valores, capacidades, habilidades y destrezas requeridas por el estudiante durante el trayecto en su ciclo básico y de especialidad.

Visto así, la investigación ha sido considerada como una competencia genérica o general que transversaliza el proceso de formación profesional en el contexto tecnológico, situación que se evidencia en los diseños curriculares de las universidades públicas y privadas de la Subregión Costa Oriental del Lago (COL), como en el caso de la Universidad del Zulia y el Instituto Universitario Politécnico Santiago Mariño extensión COL Cabimas.

De ahí que el término competencias investigativas en el campo de las ciencias aplicadas según Atencio (2014).

Se asocia con la actuación integral asumida por los estudiantes de programas académicos de naturaleza tecnológica, la cual se identifica con la capacidad del ser humano para afrontar y resolver los problemas del contexto, mediante la generación de procesos de mejoras, propuestas (modelos, metodologías y sistemas) y visibilidad del conocimiento científico, para trascender la brecha existente entre el sector tecnológico e industrial, proporcionando bienestar común para el colectivo en general.

En este sentido, resulto imprescindible analizar dichas competencias investigativas formuladas en los perfiles académicos profesionales de las instituciones universitarias mencionadas con anterioridad, en el marco de las políticas públicas 
de ciencia, tecnología e innovación prescritas por el estado venezolano, a fin de develar la pertinencia de las mismas frente a la satisfacción de necesidades del tejido social.

En torno a las políticas públicas, Atencio (2017), indica que su análisis tiene como objeto de estudio el conjunto de dispositivos conformado por: los objetivos colectivos que el Estado considera como deseable o necesarios, incluyendo el proceso de definición y de formación de estos; los medios y acciones procesadoras, total o parcialmente, por una institución u organización gubernamental; y los resultados de estas acciones, incluyendo tanto las consecuencias deseadas como las imprevistas.

En palabras del mismo autor, las políticas públicas en el campo de la ciencia, tecnología e innovación, se precisan como:

Un dispositivo articulador de esfuerzos públicos orientados a la formulación, ejecución y evaluación de proyectos y programas que buscan satisfacer las necesidades comunes de la región, mediante la armonización de herramientas e instrumentos si se quieren legales centradas en la participación colectiva de los actores sociales, para el fortalecimiento de la infraestructura científica y tecnológica, vinculando su compromiso con la sociedad, el ambiente, el crecimiento económico y la estructura de organización implícita en la gestión pública local con miras al desarrollo (Atencio, 2017).

Partiendo de lo anterior, Sánchez (2003), refiere que desde la creación del antiguo Concejo Nacional de Investigaciones Científicas y Tecnológicas (CONICIT), se asumió la agenda, como un instrumento para el diseño y la formulación de una política pública interactiva para la generación de conocimientos, tecnologías e innovación, con miras de dar soluciones a problemáticas complejas de carácter socioeconómico, político y ambiental a través de la investigación.

En este orden de ideas, se tiene que el antiguo Ministerio de Poder Popular para Ciencia, Tecnología e Innovación desde el año de su creación (2001) hasta hoy día desde su unificación con el Ministerio del Poder Popular para la Educación Universitaria, ha venido delineando diversas políticas orientadas a fortalecer el Sistema Nacional que rige la actividad científica, tecnológica y de innovación en Venezuela, procurando masificar el conocimiento y los saberes populares para la suprema felicidad y el logro de la independencia tecnológica, enmarcándose en los planteamientos del Plan Nacional de Ciencia, Tecnología e Innovación (2005-2030), y en la Ley Orgánica de Ciencia, Tecnología e Innovación (2014).

En virtud de ello, previa revisión documental de los referentes mencionados anteriormente, se etiquetaron como categoría de análisis las siguientes políticas públicas de ciencia, tecnología e innovación: acceso universal a las tecnologías de la información; organización del poder popular para la construcción del nuevo modelo productivo socialista; apoyo a la inventiva, creatividad, innovación $\mathrm{y}$ conocimiento aplicado al servicio del pueblo, y la apropiación social de la ciencia, tecnología e innovación para satisfacer las necesidades del entorno.

\section{Metodología}

La investigación se abordó mediante una tipología analítica desde un enfoque cualitativo con diseño documental bibliográfico, partiendo de la revisión de textos escritos (Informes de gestión, libros, documentos online, reglamentos, entre otros) que se asocian a la problemática tratada, lo que permitió derivar categorías y subcategorias orientadas a la comprensión de las competencias del investigador en el contexto tecnológico y su correspondencia con las políticas públicas venezolanas de ciencia, tecnología e innovación.

Como unidades de análisis se asumieron los perfiles académicos curriculares del campo tecnológico (Competencias generales), de instituciones universitarias públicas y privadas de la Costa Oriental del Lago de Maracaibo (Universidad del Zulia e Instituto Universitario Politécnico Santiago Mariño, extensión COL Cabimas), al igual que documentos que orientan el Sistema Nacional de Ciencia, Tecnología e Innovación en Venezuela; lo que implicó el diseño de una matriz de registro como instrumento de recolección de datos, apoyado en el uso de la técnica de revisión documental. En cuanto al tratamiento de los datos, se asumió la técnica de análisis de contenido sobre la base de una triangulación de fuentes, lo que permitió la contrastación de posturas sobre el fenómeno abordado, y desde esta, la interpretación de unidades discursivas que permitieron la estructuración de categorías emergentes.

\section{Resultados y discusión}




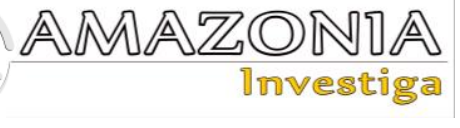

Partiendo del ejercicio hermenéutico efectuado sobre la base de los documentos que sirvieron

Tabla 1.

Matriz de Hallazgos. Competencias Investigativas en Perfiles Académicos Curriculares.

\begin{tabular}{lll}
\hline CATEGORIA DE & COMPETENCIAS GENERICAS & COMPETENCIAS GENERALES IUPSM \\
ANALISIS & LUZ (2007) & COL (2011) \\
\hline
\end{tabular}

Las Tecnologías de Información, se contemplan como una competencia genérica desvinculada de la investigación, aun cuando se presencia que para dicho proceso se requiere que del acceso y dominio de las misma,

Acceso universal a las Tecnologías de la Información dado que se busca que el futuro profesional maneje fuentes de información documentales, para que posteriormente logre discriminar dichas fuentes.

Las competencias genéricas de LUZ, requieren que el futuro profesional lidere grupos de trabajo para integrar esfuerzos, y de tal manera, establecer vínculos y relaciones con el objeto de estudio, reflexionando sobre la realidad

nuevo modelo productivo socialista

social y el ambiente que lo rodea para transformarlo; lo que le conlleva a gestionar procedimientos para la producción de cambios en su entorno, y de tal manera, lograr explicar y comprender los hecho y fenómenos espacio-temporales.

Solo se hace énfasis en la producción del conocimiento bajo un esquema lineal, mediante la observación, descripción, explicación y predicción. Es de suma importancia señalar, que las competencias genéricas analizadas obvian en su totalidad los procesos vinculados con la investigación tecnológica, y sus productos derivados, entre ellos: diseño de prototipos, sistemas o modelos; registro de patentes, escalamientos industriales y la creatividad, inventiva e innovación como procesos que se complementan. Se presencian competencias orientadas a valorar la función social de la investigación; sin embargo, no se evidencian competencias para la divulgación y promoción de la ciencia tecnología e innovación en espacios de reflexión comunitaria, como mecanismo para la apropiación social del conocimiento y tecnologías generadas. entorno como unidad de análisis, se obtuvieron los siguientes hallazgos COL (2011)

La competencia de Investigación, Innovación y Desarrollo, se deslinda del uso de las tecnologías de la información para el abordaje del proceso metodológico en su carácter tecnológico, aun cuando, es preciso notificar que las competencias generales del egresado de esta institución, reconoce la tecnología de la información y comunicación como otra competencia fuera de la analizada.

Se manifiestan criterios de desempeño actitudinal que implican demostrar la capacidad del egresado para tomar en cuenta las necesidades y exigencias presentes en el entorno en la creación de ideas o productos innovadores partiendo del trabajo colectivo. Sumado a ello, se refleja que el futuro profesional debe demostrar competencias para motivar a su equipo a participar proporcionando ideas innovadoras. Visto así, es preciso acotar, que no existen criterios de desempeños conceptuales y procedimentales orientados a fortalecer la política categoría de análisis.

En un sentido de totalidad se presencian criterios de desempeños que conllevan al futuro profesional demostrar capacidades para desarrolla productos, técnicas, herramientas, sistemas, cultura o servicios novedosos, útiles y competitivos, en los cuales se trabaja a partir de una idea generada por necesidades detectadas en el entorno, tomando en cuenta sugerencias, innovando y fomentando la participación proactiva y el liderazgo eficaz. Sumado a ello, se hace énfasis en la producción de conocimiento para generar bienestar al colectivo social.

Se hace énfasis en competencias que implican demostrar dominio y seguridad del futuro profesional ante la socialización de los hallazgos derivados del estudio realizado, reconociendo el de la investigación como fuente generadora de nuevos conocimientos. Sin embargo, no se evidencian competencias orientadas a la vinculación social con las comunidades.

innovación, cobra importancia la formación del talento humano, espacio que tal vez se podrá visibilizarse desde el desarrollo de competencias en quienes hacen vida académica en los diversos recintos universitarios, mediante modelos ha asignado mayor relevancia a los procesos vinculados con la ciencia, tecnología e 
curriculares adaptados a las necesidades del entorno.

Por su parte, la mirada es imperante volver la mirada a los planteamientos de Inciarte $\mathrm{y}$ Canquiz (2008), quienes refieren que uno de los retos fundamentales, que ya se han venido delineando es la integralidad en la formación, la cual solo será posible en la medida que se dé la pertinencia, entendida esta como la repuesta filosófica, científica, social, personal, laboral e institucional. Siendo así, se estima entonces que las competencias del investigador en el contexto tecnológico son poco pertinentes al obviar requerimientos específicos que desde la política nacional se orientan al desarrollo e innovación tecnológica como mecanismo para el crecimiento socioeconómico nacional. A continuación se muestra el abordaje cualitativo que desde la triangulación de fuentes sintetiza las categorías emergentes que encuentran lugar desde el ejercicio de hermeneusis documental emprendido.

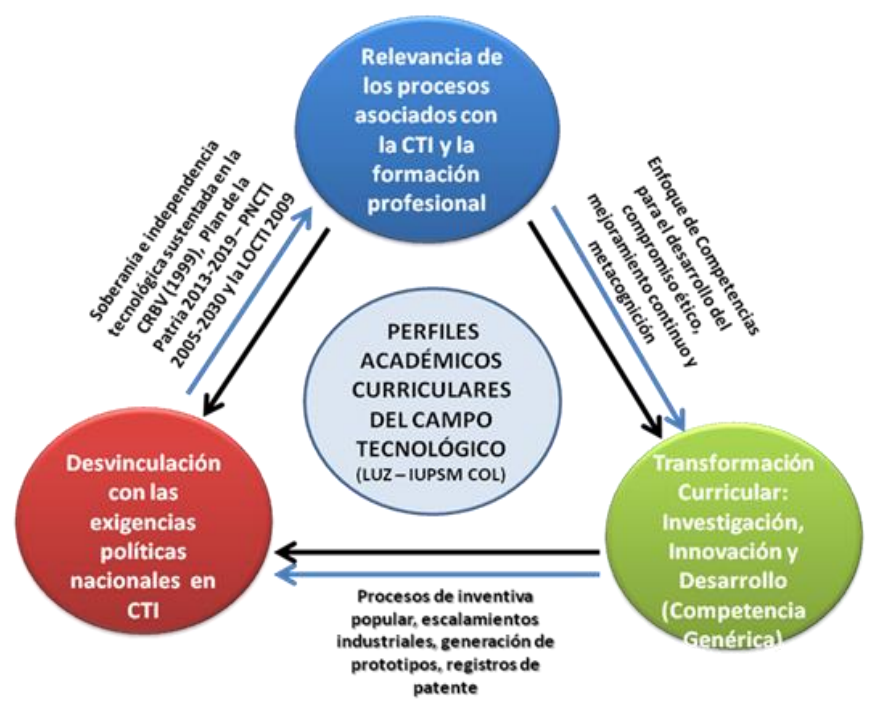

Figura 1. Graficación del Abordaje Cualitativo. Fuente: Elaboración propia (2021)

\section{Conclusiones}

Partiendo del análisis contentivo en la matriz de hallazgos emergentes, se puede decir que existe una escueta correspondencia entre las competencias del investigador del contexto tecnológico estudiado y las políticas públicas de ciencia, tecnología e innovación analizadas, lo que implica profundizar en criterios de desempeño orientados al acceso universal a las tecnologías de la información, construcción del nuevo modelo productivo socialista, inventiva, creatividad, innovación y conocimiento aplicado al servicio del pueblo, y por ende a la apropiación social de la ciencia, tecnología e innovación para satisfacer las necesidades del entorno, con mayores niveles de congruencia desde la declaración que se precisa en los perfiles académicos de competencias generales contemplados como unidades de análisis.

Visto desde la perspectiva del perfil académico curricular de la Universidad del Zulia (2007), se evidenció que existe una parcial desvinculación de las Tecnologías de la Información con las competencias investigativas que se pretenden desarrollar durante el ciclo de formación profesional, aun cuando dichas competencias proporcionan la construcción del nuevo modelo productivo socialista nacional, a pesar de que se obvia la inventiva, creatividad e innovación como mecanismos articuladores entre la ciencia y la tecnología, procesos que no refieren criterios de desempeños para su promoción y divulgación. No obstante, desde la intencionalidad curricular parece estar implícito, pero no concreto para su operacionalización desde los espacios formativos contemplando para tal fin.

Por otro lado, se tiene que el perfil académico de egreso del Instituto Universitario Politécnico Santiago Mariño - COL Cabima (2011), reporta competencias fundamentadas en el desarrollo del uso y dominio de la tecnología de la información y comunicación aislada de la competencia genérica de investigación, aun cuando el perfil analizado se destina a la construcción del nuevo modelo productivo socialista, precisando indicadores de desempeño conceptuales, actitudinales y procedimentales para el desarrollo del potencial creativo, inventivo e innovador, 


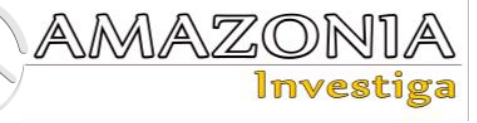

conducentes a la promoción y divulgación de los hallazgos determinados sobre la realidad.

De todo lo anterior se puede contemplar como un gran punto de reflexión y convergencia, los esfuerzos curriculares que las universidades de la Sub Región Costa Oriental del Lago-Estado Zulia, Venezuela, han consumado a favor del desarrollo integral del futuro profesional del campo tecnológico, pero aun siendo así, se amerita de una alineación curricular que dé lugar a la innovación del currículo frente a las nuevas exigencias del contexto social y ocupacional, sin dejar de poner al estudiante como actor protagónico que se sitúa al centro de toda la dinámica de la vida universitaria.

\section{Referentes Bibliográficas}

Atencio, E. (2014). Competencias investigativas con énfasis en el campo tecnológico en estudiantes universitarios. REDHECS: Revista electrónica de Humanidades, Educación y Comunicación Social, 9(18), 143-161.

Atencio, E. (2014). Generación de competencias investigativas con énfasis en el campo tecnológico para estudiantes universitarios (Tesis de maestría). Universidad Nacional Experimental Rafael María Baralt. Venezuela. Recuperado de: https://www.academia.edu/41958049/TESIS_DE_ MAESTR\%C3\%8DA_GENERACI\%C3\%93N_D E_COMPETENCIAS_INVESTIGATIVAS_CON _C3\%89NFASIS_EN_EL_CAMPO_TECNOL \%C3\%93GICO_PARA_ESTUDIANTES_UNIVE RSITARIOS

Atencio, E. (2017). Políticas públicas de ciencia, tecnología e innovación. Aproximaciones latinoamericanas. Ciudad Maracaibo: Editorial Astro data.

Bozo de Carmona, J., Camacho, H., Carillo, G., Casilla, D., Inciarte, A., Morales A., Rincones, L., \& Parra, M. (2009). Proceso de Bolonia en América Latina. Caso Venezuela. Resumen provisorio "Proceso de Bolonia en la Educación Superior en América Latina", Venezuela, Observatorio Internacional de Reformas Universitarias (ORUS). Recuperado de: http://www.slideshare.net/AsambleaESAP/elproceso-de-bolonia-en-la-educacion-superior-deamerica-latina

Castillo, S. \& Cabrerizo, J. (2006). Formación del profesorado en educación superior. Didáctica y Curriculum. España: Editorial McGraw-Hill.
Decreto de rango, valor y fuerza de Ley Orgánica de Ciencia, Tecnología e Innovación. Gaceta Oficial Extraordinaria $\mathrm{N}^{\circ}$ 6.151, Venezuela, 20092014.

Decreto $\mathrm{N}^{\circ}$ 1.411. Decreto con rango, valor y fuerza de Ley de Ciencia, Tecnología e Innovación. Gaceta oficial de la República Bolivariana de Venezuela, Caracas, Venezuela, 13 de Noviembre de 2014. Recuperado de: http://extwprlegs1.fao.org/docs/pdf/ven147980.pdf Inciarte, A，\& Canquiz, L. (2008). Formación integral desde el enfoque por competencias. Venezuela, Colección Textos Universitarios: Ediciones del Vice rectorado Académico de la Universidad del Zulia.

Instituto Universitario Politécnico Santiago Mariño COL Cabimas (2011). Competencias generales del egresado, Venezuela, Material mimeografiado. Recuperado de: https://www.academia.edu/50172048/PERFIL_AC AD\%C3\%89MICO_PROFESIONAL_DEL_EGR ESADO_DEL_IUPSM_COMPETENCIAS_GEN ERALES

Ministerio del Poder Popular para la Ciencia y Tecnología de Venezuela (2011). Necesidad de investigación 2011. Recuperado de: http://www.ucv.ve/fileadmin/user_upload/facultad _humanidades/documentos/coordinacion_de_inves tigacion/2011/31.01.2011_necesidades_investigaci on_mppctii_1_.pdf

Perilla, J. (Ed.). (2018). Diseño curricular y transformación de contextos educativos desde experiencias concretas. Ciudad Bogotá: Editorial Universidad Sergio Arboleda.

Rincones, L. (2007). El Currículo Cooperativo Incardinado. Trama Teórica de Sustentación, (Tesis doctoral), Universidad del Zulia, Facultad de Humanidades y Educación. División de Postgrado, Venezuela.

Sánchez. (2003). Política pública en ciencia y tecnología: las agendas de Conicit. Ciudad Caracas: Editorial CEDES.

Tobón, S. (2010). Formación integral y Competencias: Pensamiento complejo, currículo, didáctica y evaluación. Colombia. 3ra edición. ECOE Ediciones.

Universidad del Zulia (2007). Perfil de Competencias Genéricas, Venezuela, Material mimeografiado. Recuperado de: https://curriculoluzcol.files.wordpress.com/2015/0 2/competencias_generales_mayo_2007.pdf 